\title{
THE ACM93 SMALL BODIES DATA BASES
}

The papers in this section present data bases on the solar system small bodies. Th idea was not to create a single centralized data base, not even a single provider of th data. Centralized data bases run the risk of being too rigid, and therefore of becomin, very quickly obsolete. On the contrary in a such a rapidly moving field of research th guarantee that the data bases will be continously updated is more important than th guarantee of stability of the data. Therefore we are here only providing the informatios on the existence of data bases which are maintained and upgraded by the people whr created them. The only requirement we have enforced is that the data have to br available to everybody, either by a self service system (such as anonymous $\mathrm{ftp}$ on th. internet computer network), or upon request (to be forwarded by either E-Mail o ordinary mail). This commitment to availability has been accepted by all the provider of the data bases listed in the following papers; moreover, the service of giving acces to the data cannot be a commercial entreprise, although in some case the cost of th magnetic media/paper copies/electronic subscription has to be payed by the user.

The following eight papers describe, for each data base, the data sets currentl: available, the source of the data, the access method, the address of the contact person(s) as well as providing other useful information, such as references, availability of publi domain software and/or user interfaces. Some papers also include comments on th quality and reliability of the data, and predictions on their future upgrades; however more complete information has to be looked for in the data bases themselves (e.g. in README and other documentation files and in online help) and/or asked to the contac person. This is necessary not only to save space, but also because the time scales fo. upgrade of the data bases are often comparable to the time scale of the publication $o$ this book. The readers will also notice that we have given up the publication of th, printout of the data: the size of the data bases is now much bigger than the information which can be printed in a volume such as this one; the printed versions are generall! available upon request to the contact persons. 